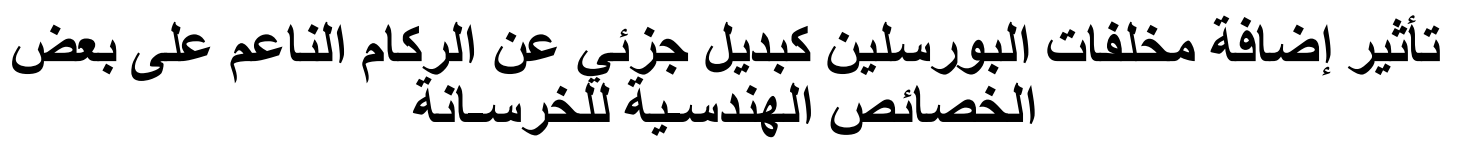

$$
\begin{aligned}
& \text { زينة عادل محمد } \\
& \text { مدرس مساعد ماله } \\
& \text { جامعة الموصل/ كلية الهندسة ـ قسم الهندسة الدنية }
\end{aligned}
$$

بدأ في السنوات الأخيرة السعي بالاهتمام وراء الحصول على مصسادر جديدة للركام الطبيعي المستخدم في الطياء

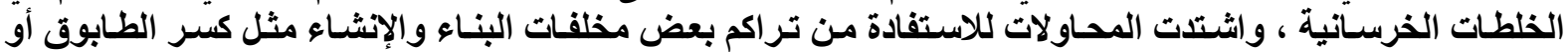

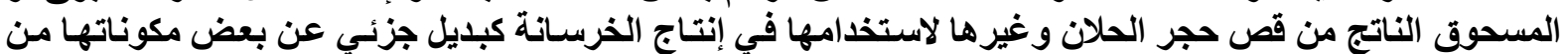

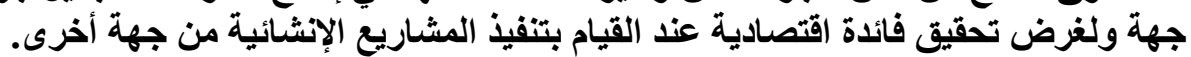

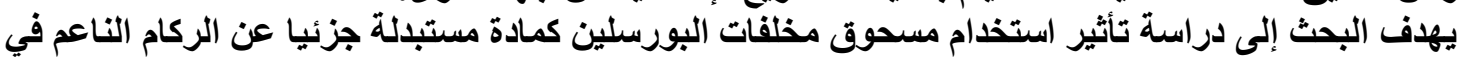

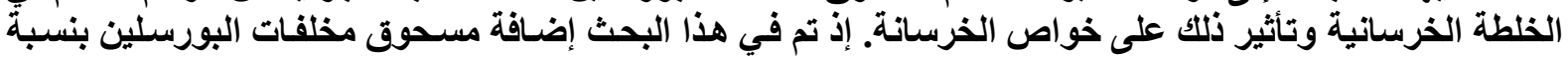

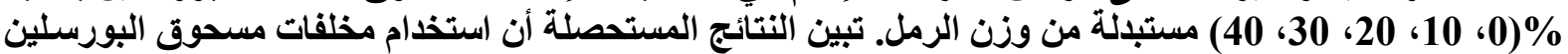

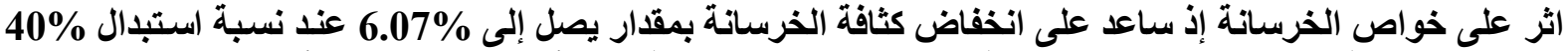

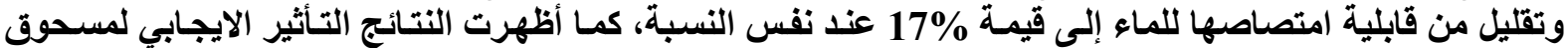
البورسلين على مقاومة الضغط والثد بزيادة تصل إلى 18\% عند نسبة استبدال 20\% مقارنة مع الخرسانة الاعتيادية.

\title{
Effect of Partial Replacement of Fine Aggregate on Some Engineering Properties of Concrete
}

\author{
Zeena Adel Mohammed \\ Assistant lecturer \\ Civil Engineering Dept./ University of Mosul
}

\section{Abstract}

Recently, interest set out for obtaining new recourses for natural aggregate ate used in concrete mixes. Attempts are also intensified to make use of the accumulation of certain types of construction and building residues such as bricks' fragments or the filler that results from the cutting of limestone and others to be used in concrete production as a partial replacement material of some of its components on the one hand and in order to achieve an economical benefit while executing the constructional projects on the other.

The research aims at studying the effect of using porcelain crushed filler as a partial replacement material for the fine aggregate in the concrete mix and the effect of this on the concrete properties. In this research, the porcelain crushed filler was added in $(0,10,20,30,40) \%$ replaced by the weight of the fine aggregate.

Findings obtained have shown that the use of the porcelain crushed filler has affected the properties of concrete as it has caused a drop in the concrete density up to $6.07 \%$ at a replacement percentage of $40 \%$ and reducing its capacity of water absorption down to $17 \%$ at the same percentage. Findings have also shown the positive effect of the porcelain crushed filler on the compressive and tensile strengths with an increase reaching $18 \%$ at a replacement percentage $20 \%$ compared to the normal concrete. 
يسعى العديد من الباحثين و الميتمين ذوي الاختصاص إلى اللجو ء لتحسين إنتاج الخرسانة بمو اصفات جيدة بالبحث

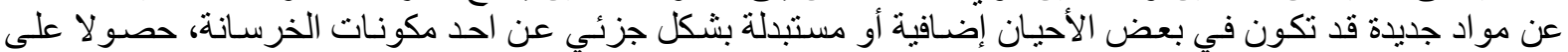

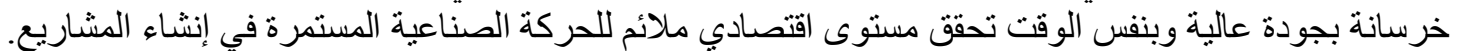

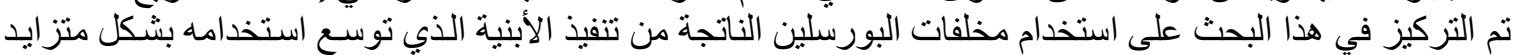

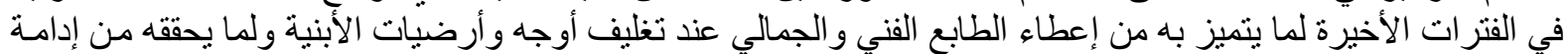

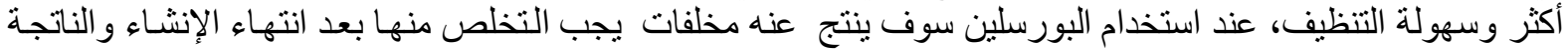

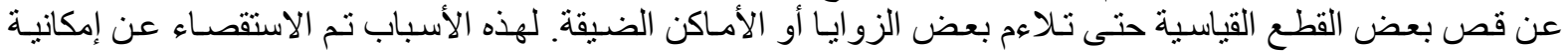

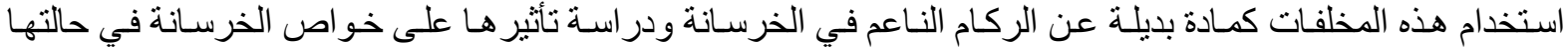
الطرية و المتصلبة.

البورسلين عبارة عن مادة بيضاء قوية تتكون من خليط من الطين النقي ، الكاؤولينات، الكوارتز (جدول

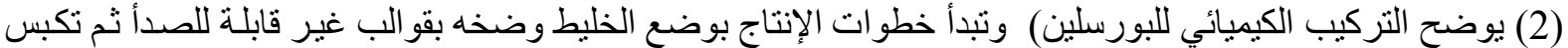

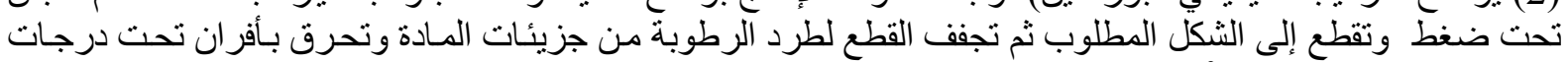

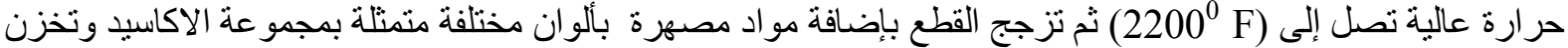

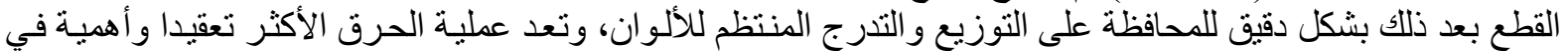

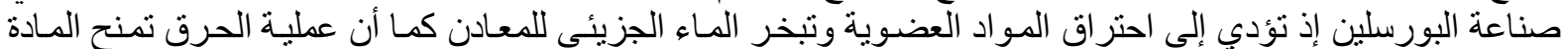

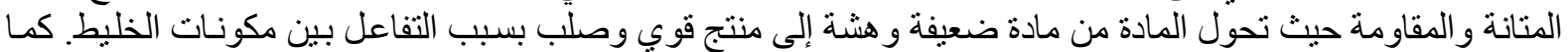

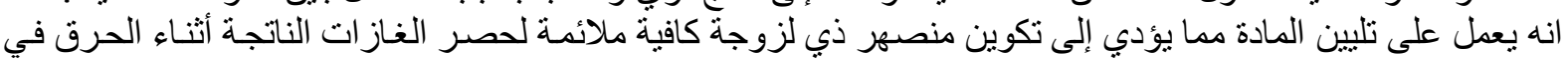

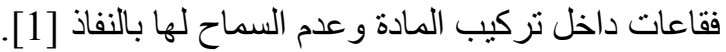

استخد/مات البورسلين

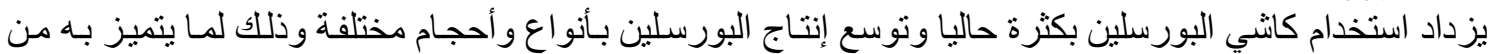

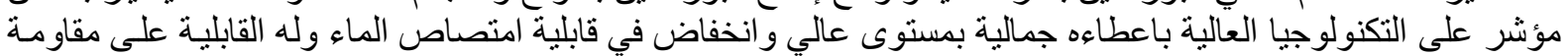

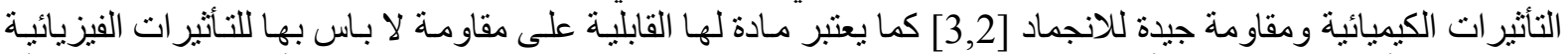

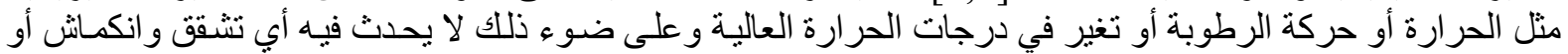

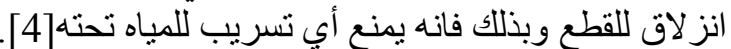

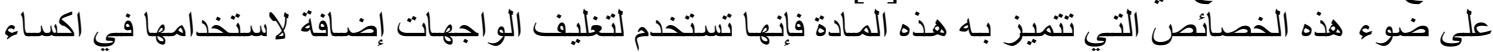

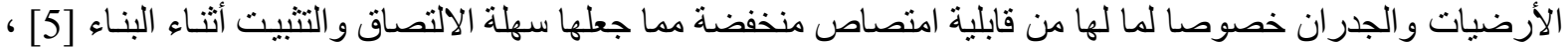

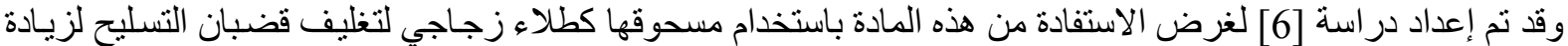

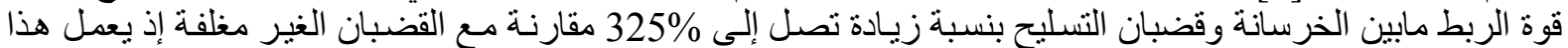

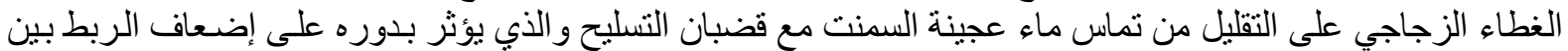

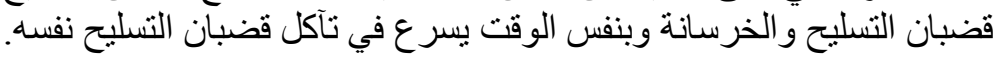

الهدف الرئيسي لهذا البحث هو معرفة تأثير إضافة مسحوق مخلفات البورسلين كبديل جزئي عن الركام

الكهدف من البحث:

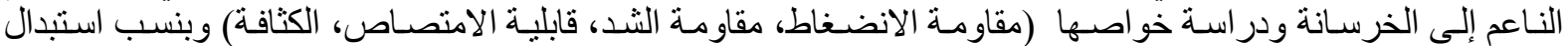

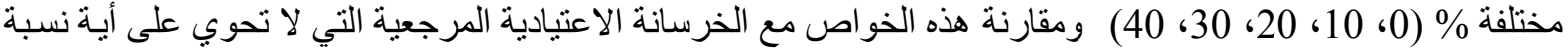

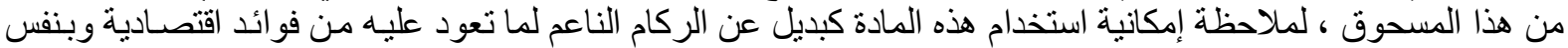
الوقت استنزاف المخلفات الناتجة من البناء و الاستفادة منها في الخلطات الخران الخانية الخانية

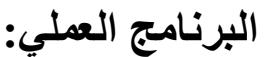

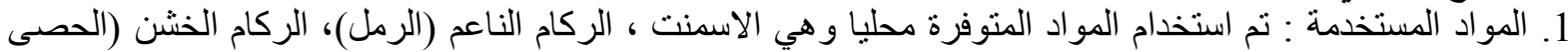

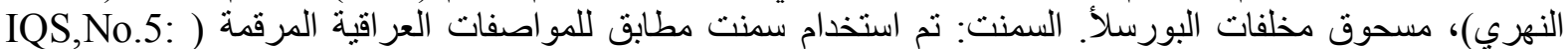
1984) [7] منتج من معمل بادوش و الجدول(1) يبين خصائص السمنت المستخدم ومقارنته مع حدود المو اصفة. 


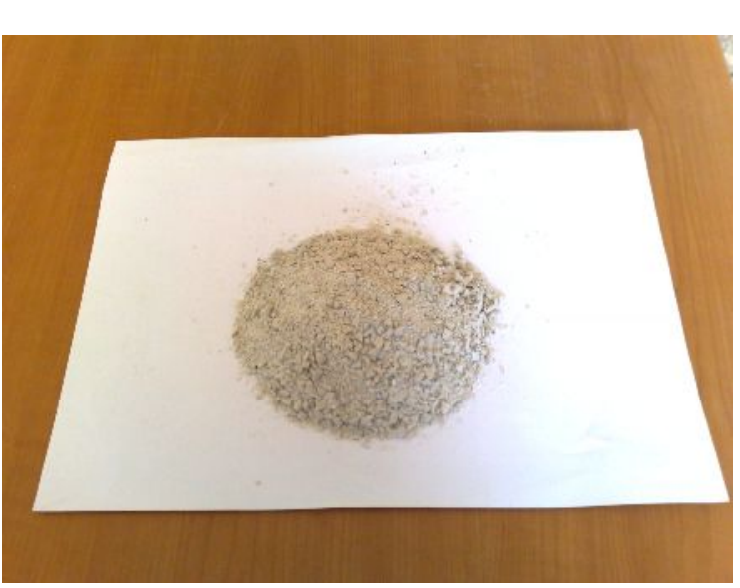

شكل (1): يوضح صورة للمادة المستبدلة ( مسحوق البورسلين)

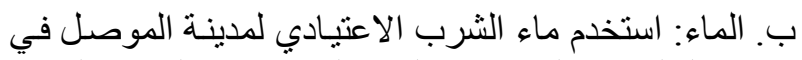

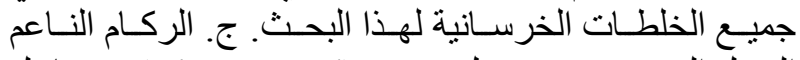
الرمل المستخدم هو رمل نهري (River Sand) وبمعامل التهل

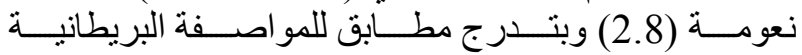
(8.S.882:1992)

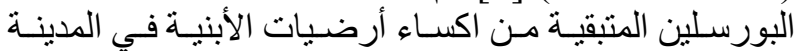

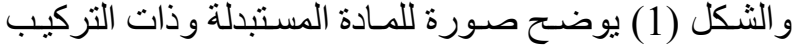
الكيميائي الموضح بالجدول (2) إذ تم طحن قطع البورسلين

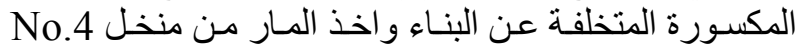

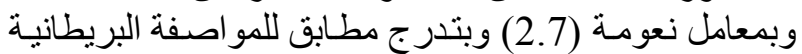

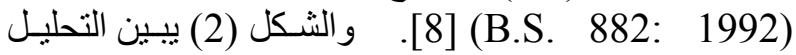
المنخلي للركام الناعم وللمادة المستبدلة.

د. الركـام الخشـن : تـم اسـتخدام الحصـى النهـري المحلـي

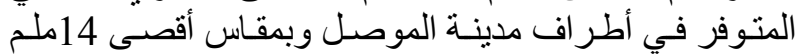
و الثكل (3) يبين تدر الريط الركام الخشن و المطـابق للمو اصفية البريطانية (3.S. 882:1992) [8. (3).

جدول (1): التحليل الكيميائي للسمنت المستخدم ومركباته الرئيسة

\begin{tabular}{|c|c|c|}
\hline حدود المواصفة (IQS,No.5: 1984) (\%) & النسبة المئوية (\%) & اكاسيد عناصر السمنت \\
\hline $3-8$ & 5.8 & $\mathrm{Ai}_{2} \mathrm{O}_{3}$ \\
\hline $17-25$ & 21.52 & $\mathrm{SiO}_{2}$ \\
\hline $0.5-6$ & 2.64 & $\mathrm{Fe}_{2} \mathrm{O}_{3}$ \\
\hline $60-67$ & 62.55 & $\mathrm{CaO}$ \\
\hline لا يزيد عن 2.8\% & 2.34 & $\mathrm{SO}_{3}$ \\
\hline 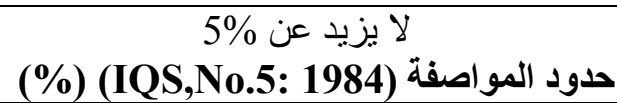 & النسبة المئوية 3.20 (\%) & $\begin{array}{c}\mathrm{MgO} \\
\text { مركبات السمنت الرئيسية }\end{array}$ \\
\hline $31.30-41.05$ & 37.02 & $\mathrm{C}_{3} \mathrm{~S}$ \\
\hline $28.61-37.90$ & 33.97 & $\mathrm{C}_{2} \mathrm{~S}$ \\
\hline $11.96-12.30$ & 12.1 & $\mathrm{C}_{3} \mathrm{~A}$ \\
\hline $7.72-8.02$ & 7.95 & $\mathrm{C}_{4} \mathrm{AF}$ \\
\hline \multicolumn{3}{|c|}{ الخصائص الفيزيائية للسمنت المستخدم } \\
\hline لا تزيد عن 10\% & $\%$ & النعومة (نسبة المتبقي على منخل رقم 170) \\
\hline \multicolumn{3}{|c|}{ وقت التماسك | } \\
\hline لا يقل عن 45 دققيقة & 135 & ابتدائي (دقيقة) \\
\hline لا يزيد عن 10 ساعات & 360 & نهائي(دقيقة) \\
\hline \multicolumn{3}{|c|}{ مقاو مة الانضغاط (MPa) } \\
\hline لا تقل عن (16 MPa) & 18 & 3 \\
\hline لا تقل عن (24 MPa) & 26 & 7 \\
\hline \multicolumn{3}{|c|}{ مقاومة الثد (MPa) } \\
\hline لا تقل عن (1.6 MPa) & 1.78 & 3 \\
\hline لا تقل عن (2.4 MPa) & 2.5 & 7 أيام \\
\hline
\end{tabular}


جدول (2): التحليل الكيميائي لمسحوق البورسلين المستخدم *

\begin{tabular}{|c|c|}
\hline (كاسيد عناصر السمنت & $\mathrm{Ai}_{2} \mathrm{O}_{3}$ \\
\hline 5.28 & $\mathrm{SiO}_{2}$ \\
\hline 15.17 & $\mathrm{Fe}_{2} \mathrm{O}_{3}$ \\
\hline 2.46 & $\mathrm{CaO}^{\prime}$ \\
\hline 53.12 & $\mathrm{SO}_{3}$ \\
\hline 1.17 & $\mathbf{M g O}$ \\
\hline 2.73 & \\
\hline
\end{tabular}

* تم التحليل في معمل سمنت حمام العليل.
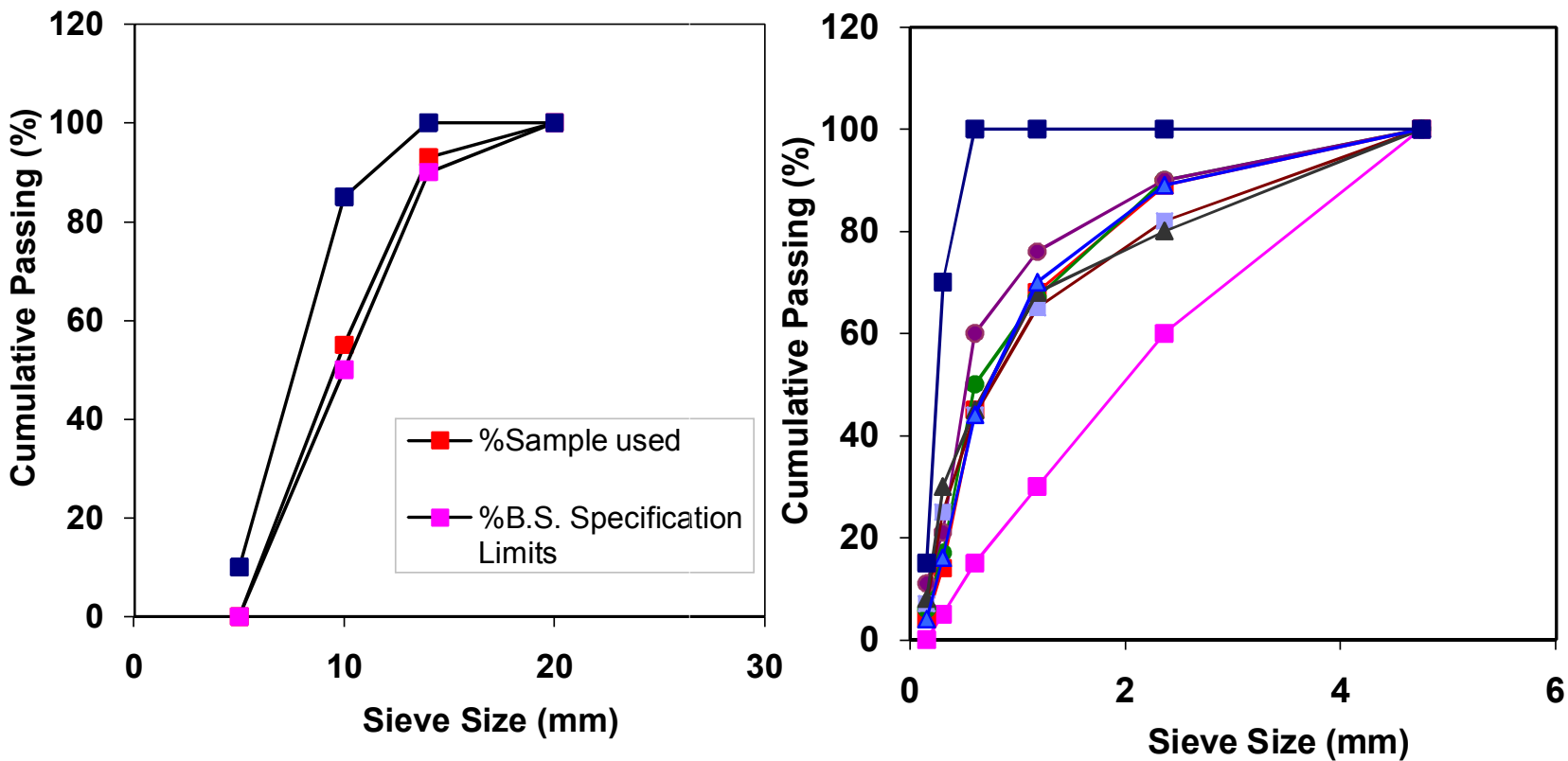

شكل (3): يوضح التحليل المنظلي للركام الخشن الخثن المستخدم مع حدود المواصفات القياسية البريطانية

(B.S. 882: 1992)

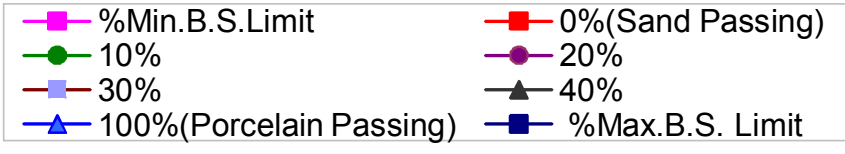

شكل (2): يوضح التحليل المنظلي للركام الناعم

ومسحوق البورسلين المستخدم مع حدود المواصفات المبلئ

القياسية البريطانية (B.S. 882: 1992)

2. نسب الخلطة الخرسانية :-

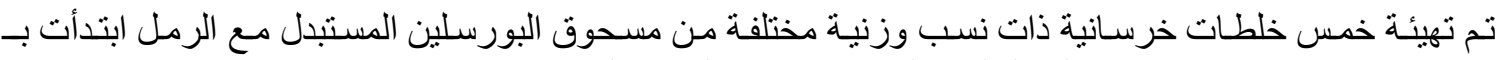

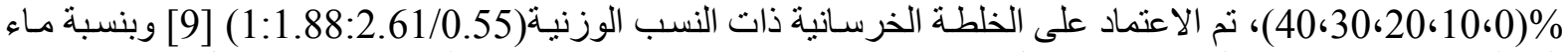

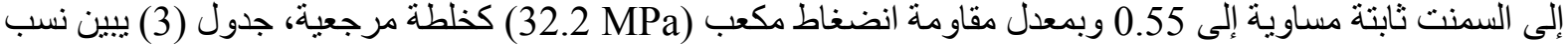

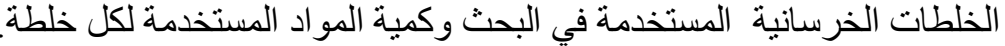

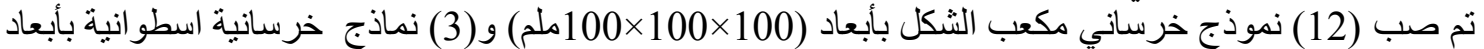

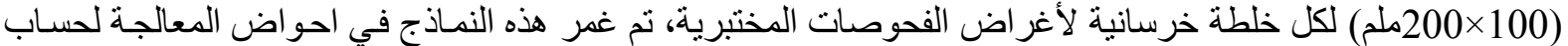

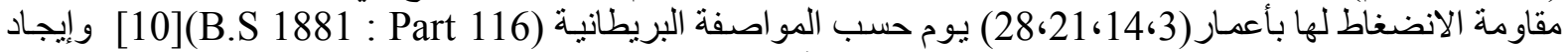

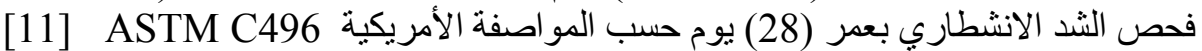


جدول (3): نسب وأوزان الخلطات الخرسانية (kg/m³)

\begin{tabular}{|c|c|c|c|c|c|c|c|c|}
\hline اللركام الناعم النعومة معادة & كغم/م3 & المستبدلة & كغم/23 & كفم/23 & كفتوى السمنت & $\begin{array}{r}\text { نسبب الاستبال } \\
\text { (\%) }\end{array}$ & النسب الوزنية & الخلطة \\
\hline 2.8 & 214.5 & 0 & 1017.9 & 733.2 & 390 & 0 & $1: 1.88: 2.61 / 0.55$ & $\mathbf{M}_{1}$ \\
\hline 2.7 & 214.5 & 73.32 & 1017.9 & 659.88 & 390 & 10 & $1: 1.88: 2.61 / 0.55$ & $\mathbf{M}_{2}$ \\
\hline 2.4 & 214.5 & 146.64 & 1017.9 & 586.56 & 390 & 20 & $1: 1.88: 2.61 / 0.55$ & $\mathbf{M}_{3}$ \\
\hline 2.8 & 214.5 & 219.9 & 1017.9 & 513.3 & 390 & 30 & $1: 1.88: 2.61 / 0.55$ & $\mathbf{M}_{4}$ \\
\hline 2.7 & 214.5 & 293.2 & 1017.9 & 440 & 390 & 40 & $1: 1.88: 2.61 / 0.55$ & $\mathbf{M}_{5}$ \\
\hline
\end{tabular}

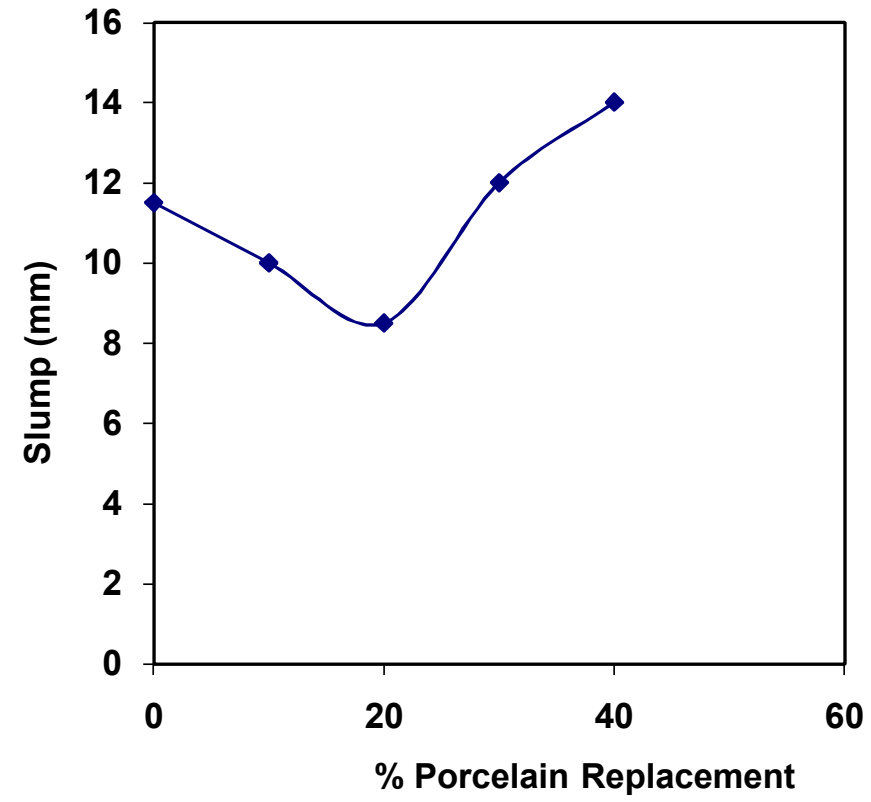

شكل (4): تأثير نسب الاستبدال المختلفة لمسحوق المولق البورسلين على الهطول

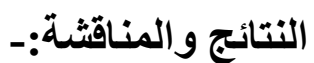

1. قابلية التثغيل:-

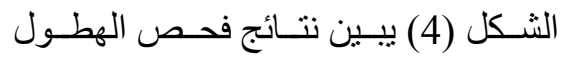

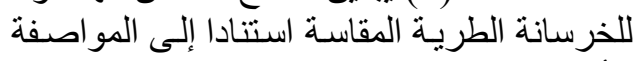

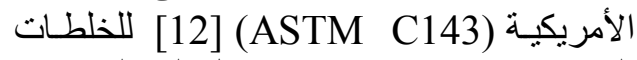

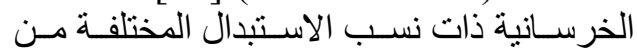

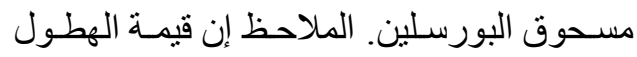

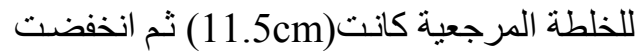

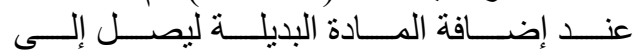
(8.5,10cm) عند نسـبة اسـتبدال (20,10\%

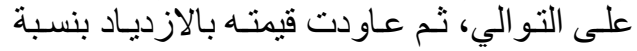
(21.73،4.3\%)

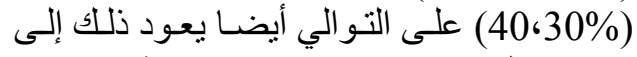

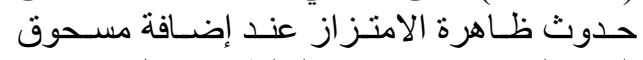
البورسلين إذ امنص ماء الخلطة من قبل جزيئات

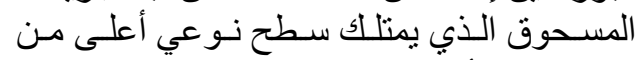

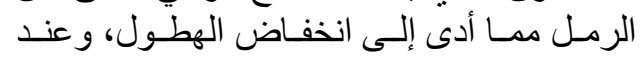

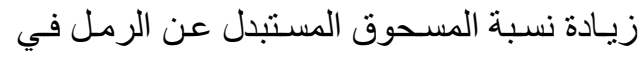

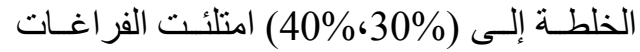

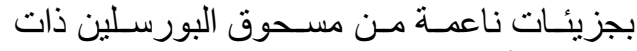

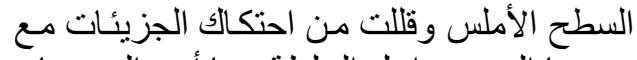

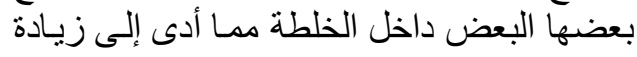
الهطول بزيادة نسبة الاستبدال. [13]

2. مقاومة الانضغاط

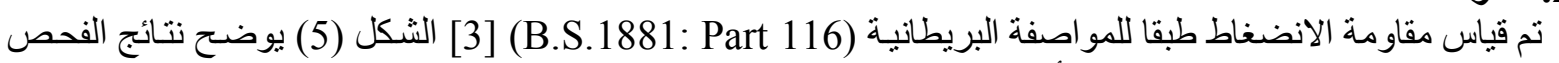

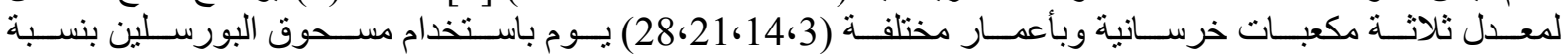

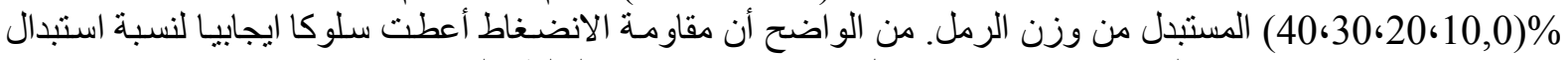
(20\%) بينما انخفضت قيمة المقاومة عند نسبة استبدال (40\%) مقارنة مع الخلطة المرجعية.

الثكل (6) بيين نمو مقاومة الانضغاط لجميع الخلطات الخرسانية وبأعمار مختلفة ويلاحظ أن هناك تأثير واضح لاستخدام مسحوق البورسلين بدلا من الرمل على نمو الانطفاط المقاومة. 


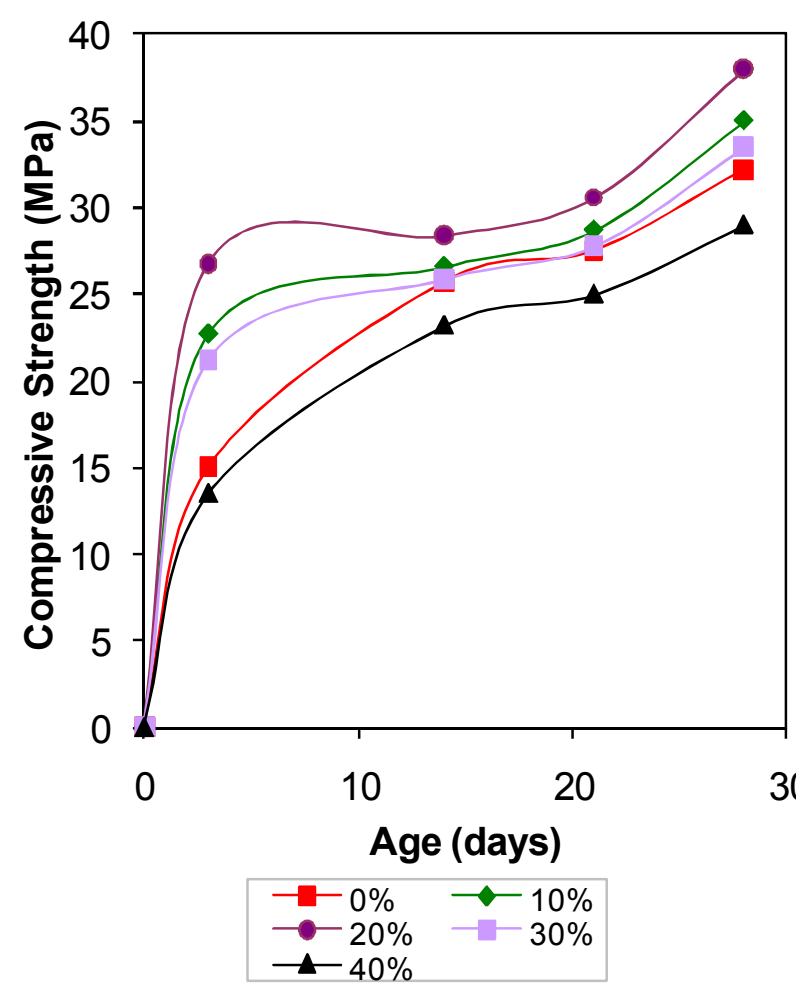

$$
\text { شكل (5): مقاومة الانضغاط للخطات الخرسانية }
$$

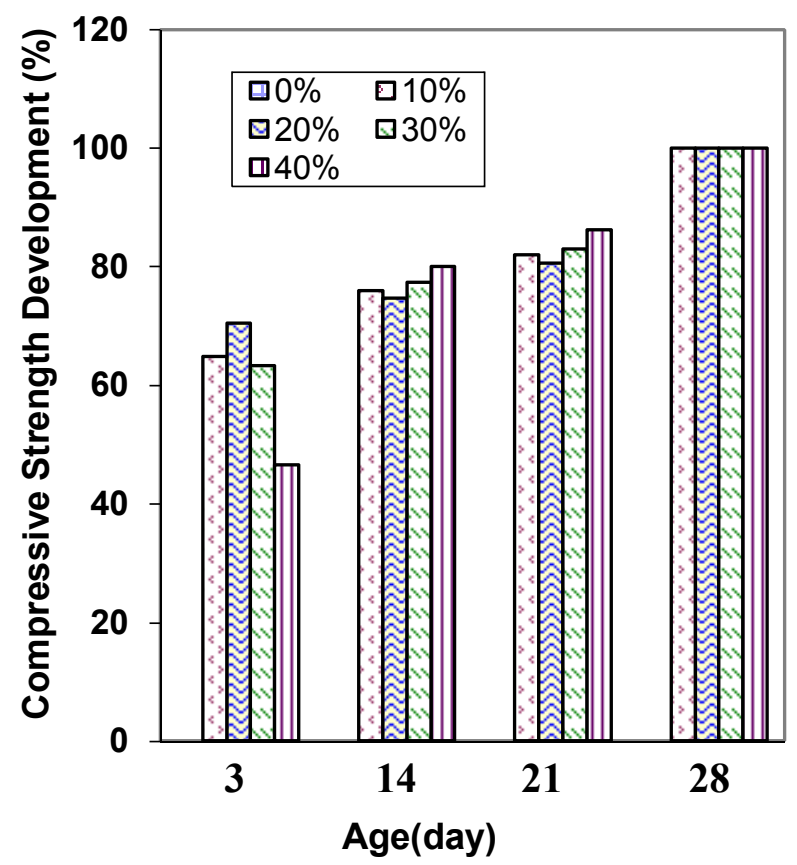

$$
\text { شكل (6): نمو مقاومة الانضغاط لجميع الخلطات }
$$

و الثكل (7) يوضح معدل مقاومة انضغاط لثناث نماذج خرسانية مكعبة عند عمر 28 يو م. وبنسب استبدال مختلفة من مسحوق البورسلين. الشكل (8) يوضح العلاقة بين مقاومة الانضغاط للخلطات الخرسانية إلى مقاومة الانضغاط للخلطة المرجعية وتأثير نسبة الاستبدال المختلفة لمسحوق البورئ لئلين.

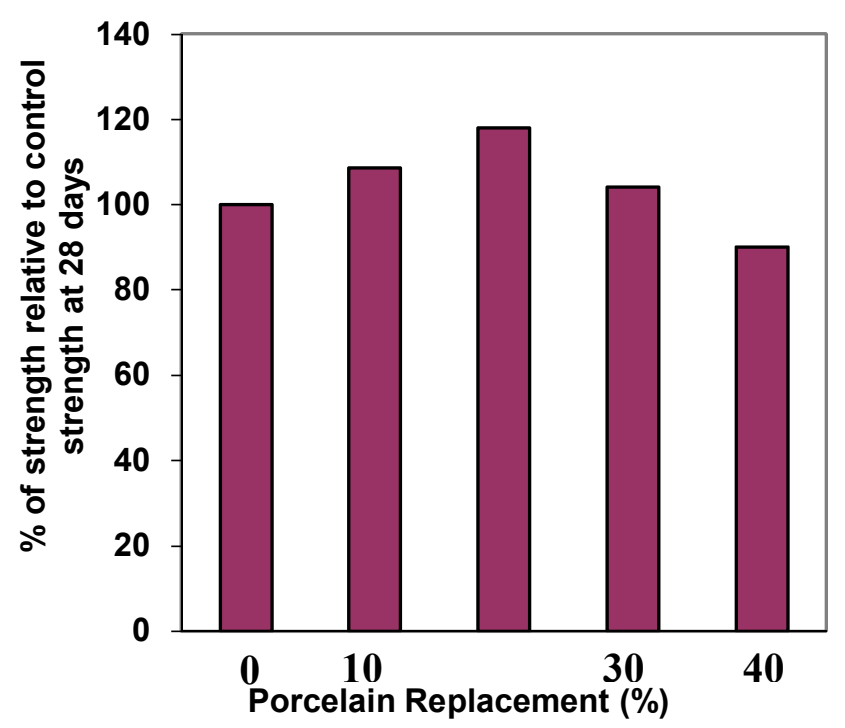

شكل (8): نمو مقاومة الانضغاط لجميع الخلطات الخرسانية وبعمر 28 يوم ئهو

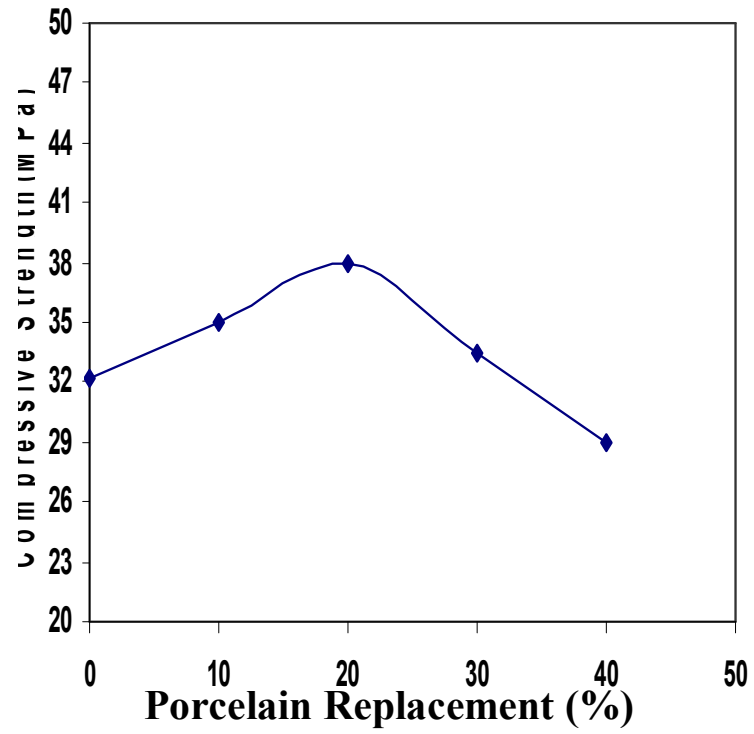

شكل (7): معدل مقاومة (نضغاط لثلاث نماذج خرسانية مكعبة ذات نسب استبدال مختلفة من مسحوق البورسلين وبعمر 28 يوم 


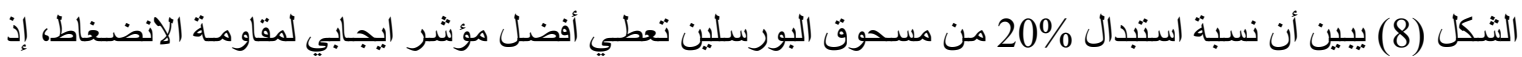

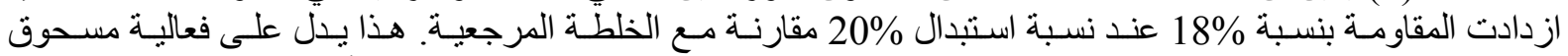

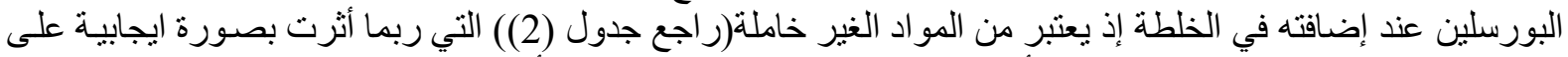

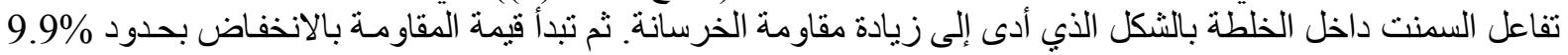

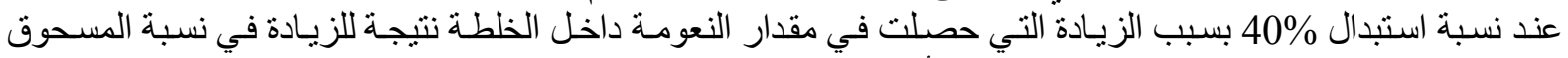

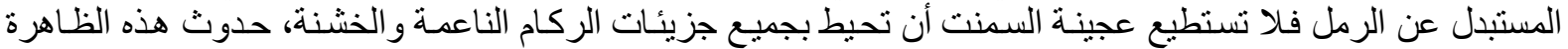
تضعف من قوة الربط بين السمنت و الركام مما أدى إلى انخفاض المقاومة بزيادة نسبة المسحوق [14].

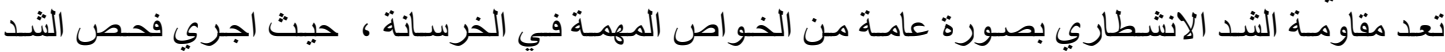

3. الثند الانشطاري:

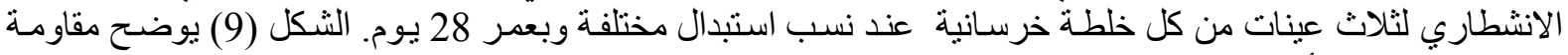
الثد الانثطاري وتأثير نسبة استبدال مسحوق البورسلين عليها.

من خلال الثكل (9) يلاحظ أن قيمة الثند الانشطاري كانت (30) (3.1 MPa) للخلطة المرجعيـة بعمر 28 يو مو وقد ازدادت

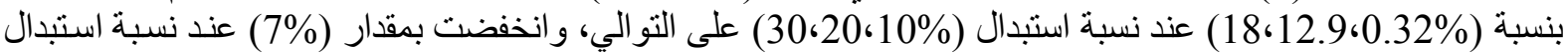
(40\%). يوضح الثكل (10) العلاقة بين النسبة المئوية لمقاومة الثد الانشطاري للخلطات الخرسية الثرانية عند نسب استبدال مختلفة من البورسلين نسبة للخر سانة المرجعية. لينة

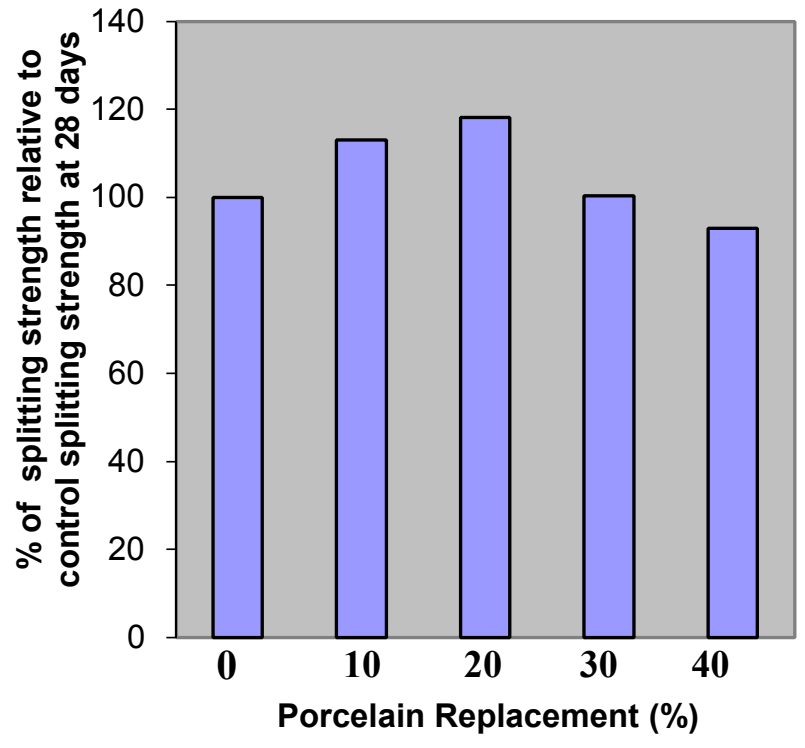

شكل (10) نمو مقاومة الثد الانشطاري لجميع

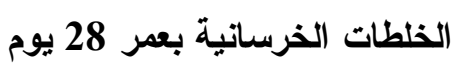

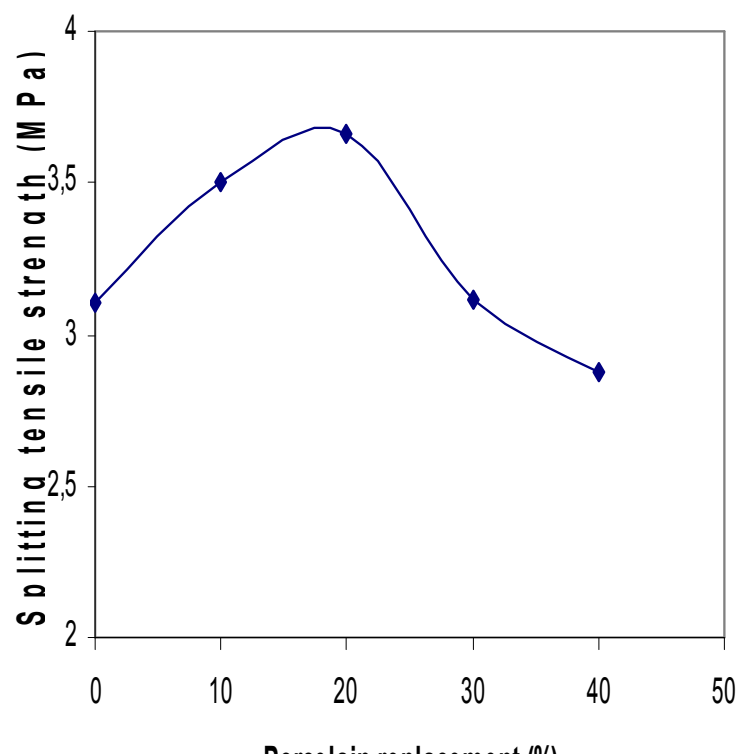

Porcelain replacement $(\%)$ الثكل(9): مقاومة الثد الانشطاري للعينات الخرسانية بعر 28 يوم

و الثكل (11) يوضح عينات فحص الثد الانشطاري وكيفية توزيع و انتشار مسحوق المادة المستبلة (البورسلين) في الخرسانة، يلاحظ من الثكل التوزيع المتجانس للمادة بين جزيئات الخرسانة وان وان نسبة التوزيع تزداد بزيادة نسبة الاستبدال

4.كثافة الخرسانة:

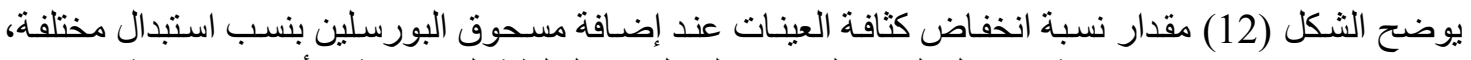

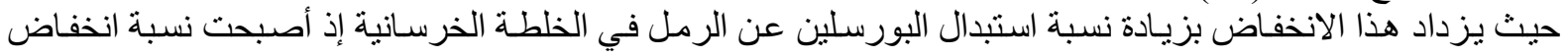

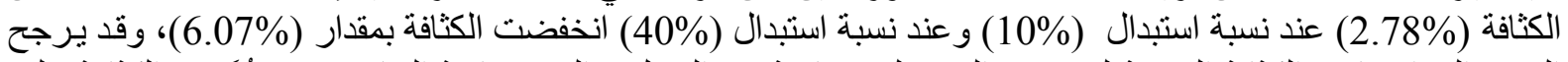

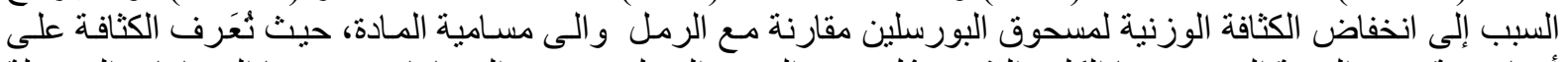

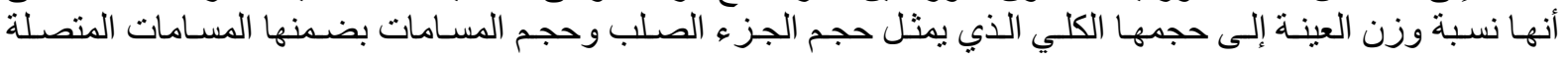
و المسامات المنفصلة وتعتمد كثافة البورسلين على حجم الحبيبات ونوزيعها ودرجة الحرق وزمنه النه عند صناعة المادة وكمية 
الغاز ات المتحررة عند الحرق و المسبية للمسامية والتزجج للمادة و التي لها تأثير على انخفاض كثافة البورسلين. النتائج في

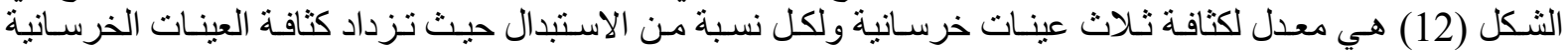

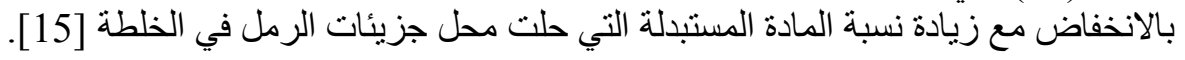

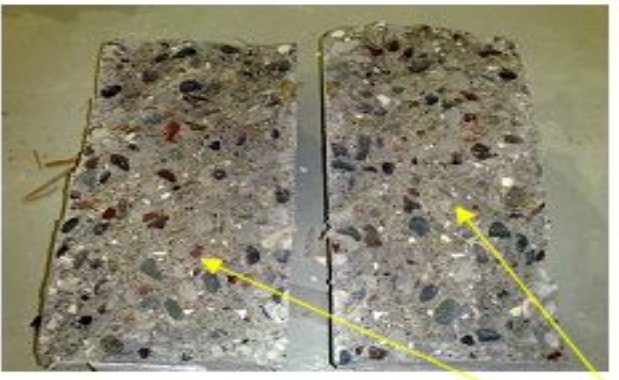

20\% تنبة أسنَبدالن

جزيئات مسحوق البورسليث (المسادة المستبلة)

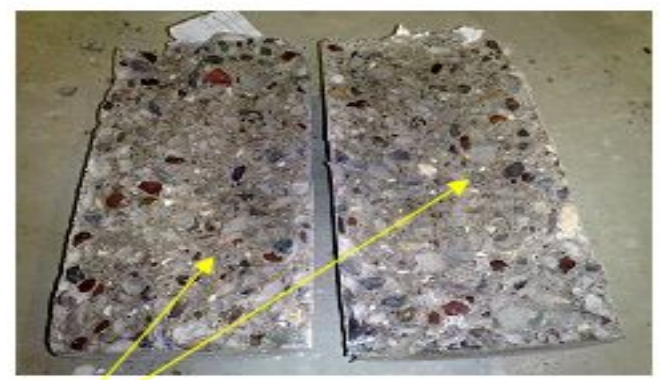

10\% نسبة المتيَدالن

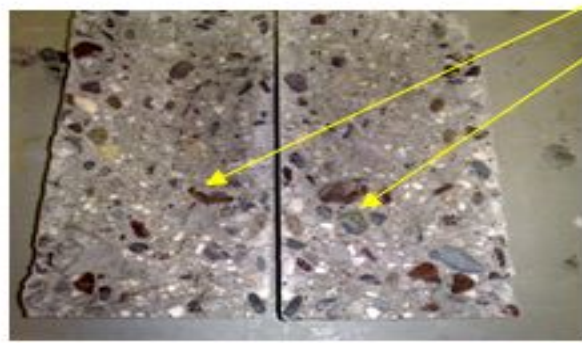

40\% تصبية الستيدالن

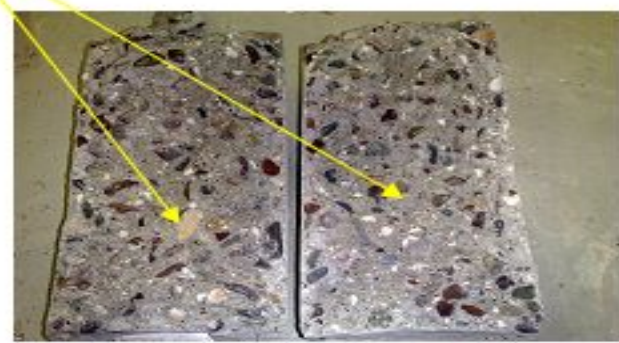

نسبة استيدالل 30\%

الثكل (11) توزيع و الثتشار جزيئات المادة المستبدلة ضمن الخرساتة

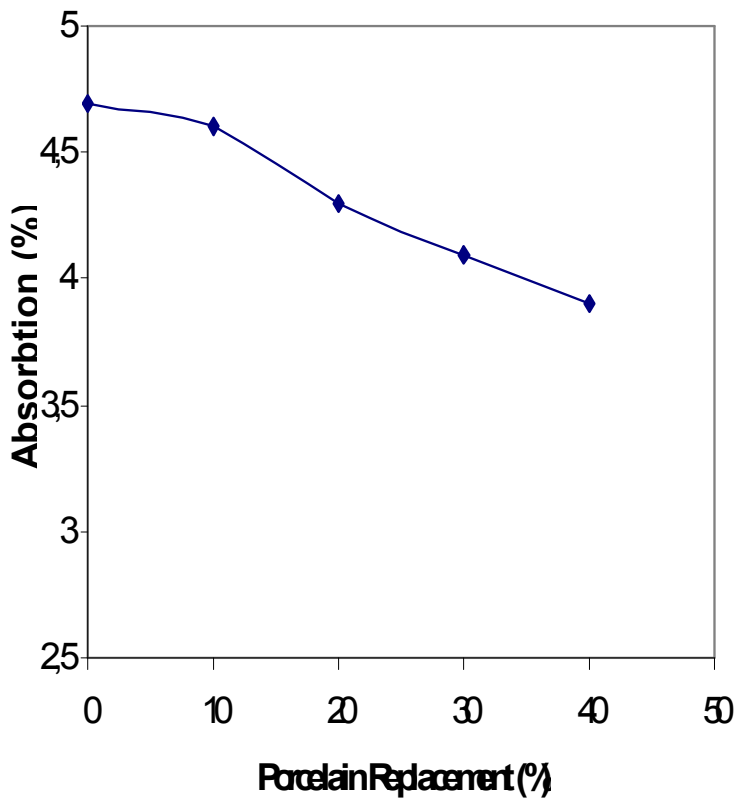

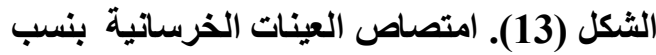

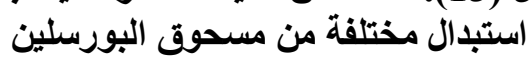

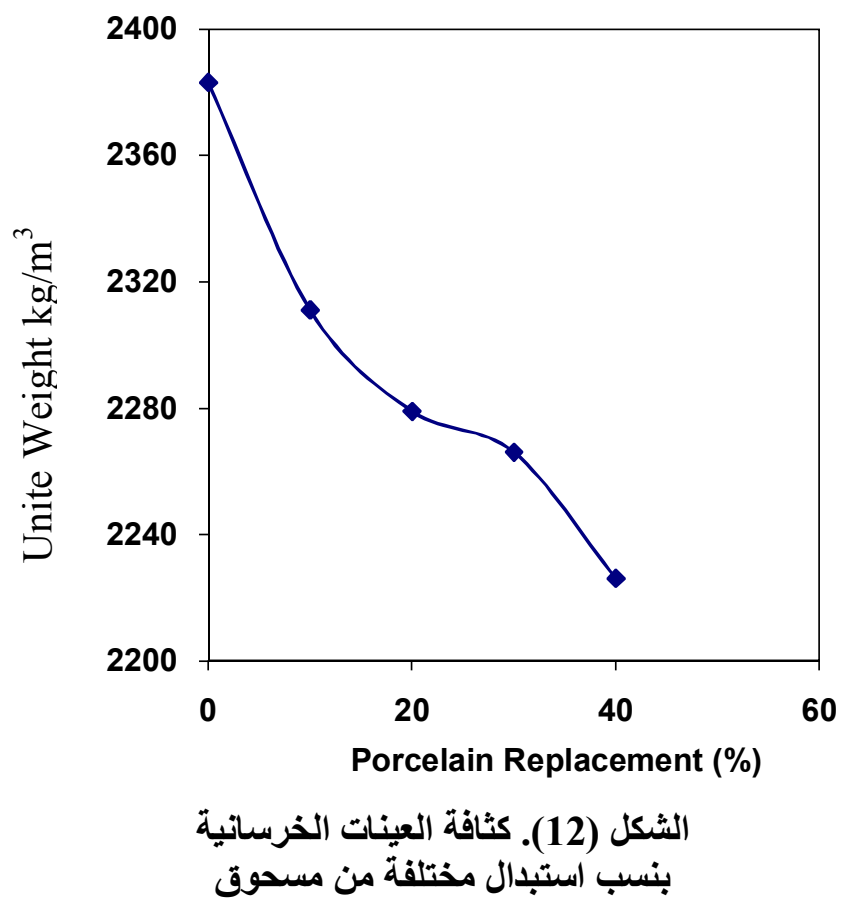


5. امتصاص العينات للماء:

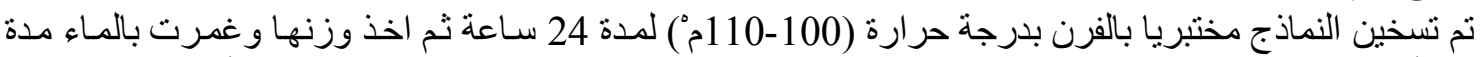

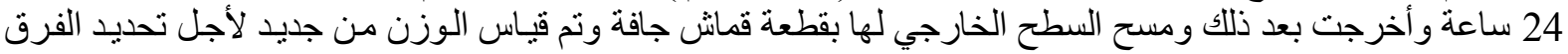

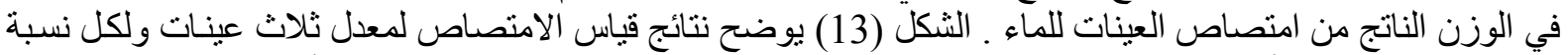

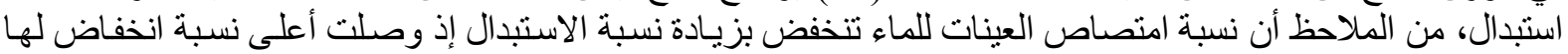

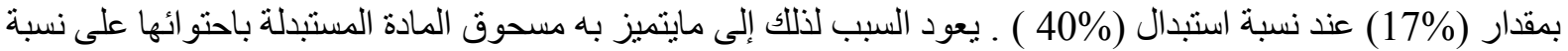

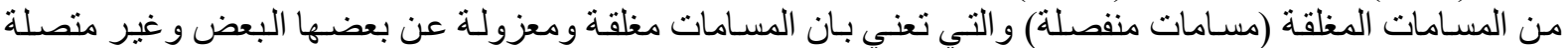

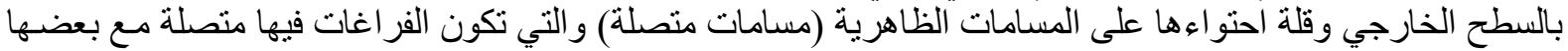

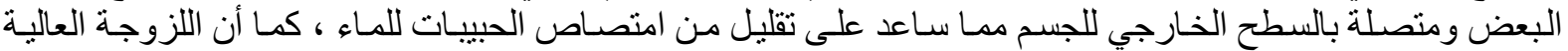

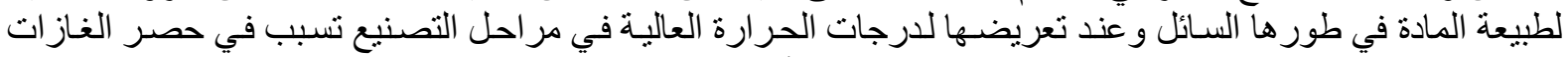

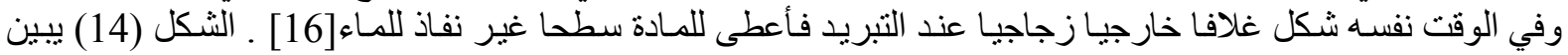
صورة مكبرة بالمجهر الضوئي (Motic Mlc- 150c) لجزيئات المادة المستبدلة المستخدمة في البحث.

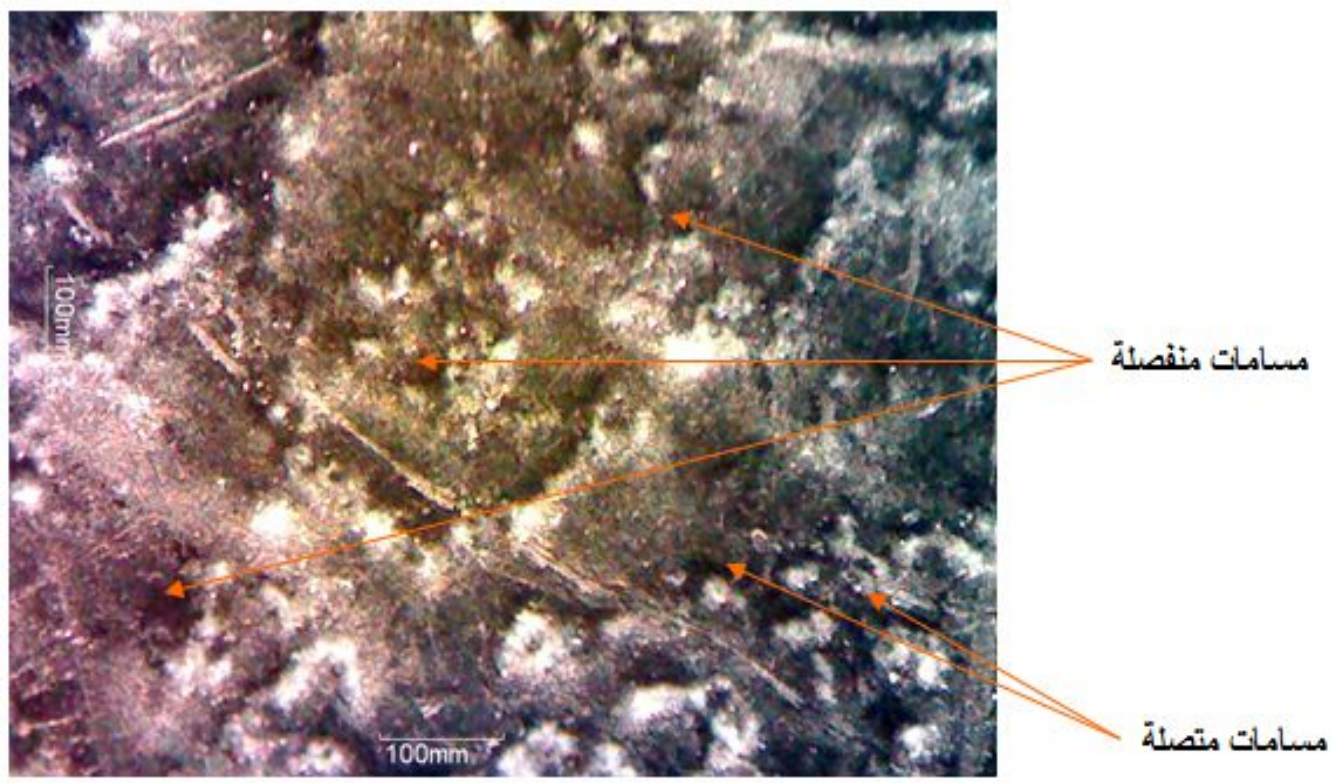

الثُكل (14): يبين صورة مكيرة لجزيئة المادة المستبلدة

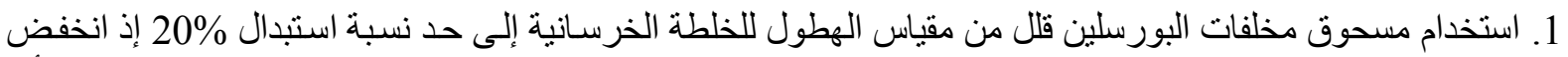

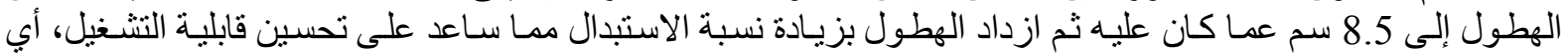

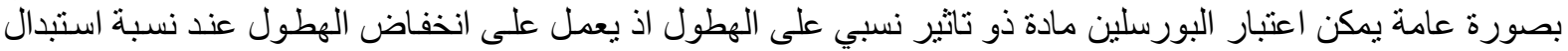
(10\%، 20\%) ويعمل على زيادته عند نسبة استبدال (30\%، (40\%).

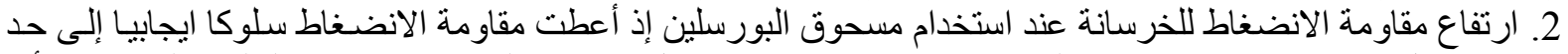

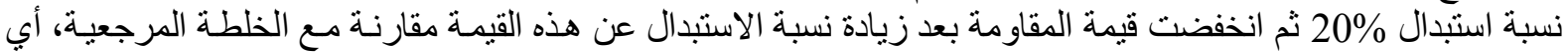

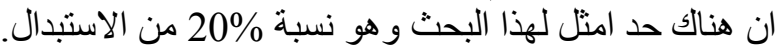

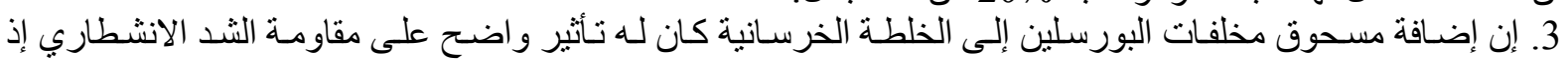

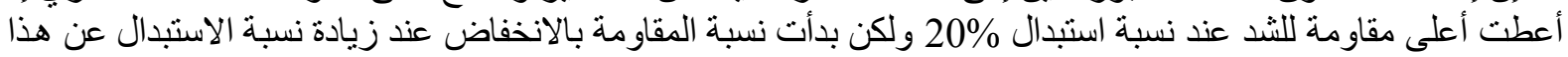
4. انخفاض كثافة العينات الخرسانية عند إضافة مسحوق البور سلين ويزداد الانخفاض بزياديادة نسبة إضـافة البورسلين في

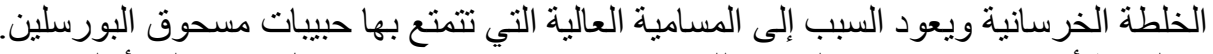

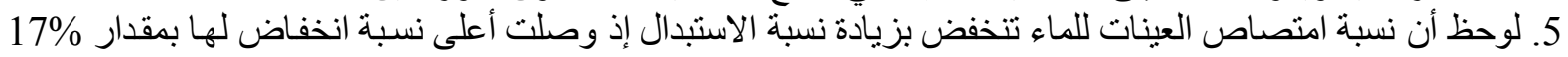
عند نسبة استبدال 40\% وذللك للطبيعة الغير نفاذة للماء التي تتميز بهاء سطح المادة المستبدلة. 
1. De' Gennaro, R., Cappelletti, P., Cerri,G., De' G ennaro,M., Dondi,M., and Langella, A., "Zeolite truffs as raw materials for light weight aggregates." Applied Clay Science, Vol.25, 2004, PP.71-81.

2.Biffi ,G.,G. ,"Porcellanto- tecnologia, produzione, mercato", Fenza, Italia :Gruppo Editoriale Fenza:, 1994.

3. Oliveira A., G., "Porcellanto: Aspectos Mercadologicos ", Tecnologicos Ceramica Industerial, Vol.3, No. 3 ,1998, PP.34-41.

4."Tile adhesive for marble, granite, ceramic and porcelain-cement based specification,", Kenya Standard, Public Review draft, Ministry of Roads and Public Works, University of Nairobi-Department of Civil Engineering, 2008, PP.1-13.

5. Almeida, A.,F., and Sichieri,E.,P., "Study of the Adherence between polymer- modified mortars and porcelain stoneware tiles",Materials Research, Vol.8,No.3, 2005, PP.245-249.

6. Hackler,C.,L., Weiss,C.,A., and Malone, P.,C.,"Reactive porcelain enamel coating for reinforcing steel to enhance the bond to concrete and reduce corrosion",International enamellers congress, Shanghai- China, May,2008, PP.18-22.

7. المو اصفات القياسية (رقم 5)، (1984) " خصائص السمنت البورتلاندي الاعتيادي"، الجهاز المركزي للتقبيس

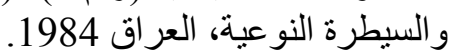

8. B.S. 882 -1992, "Aggregate from Natural Source for Concrete", British Standard Institution, 1992.

9. كثموله، سفيان يونس احمد، "تقدير مقاومة الخرسانة بعمر 28 يوم باستخدام فحص المقاومة المعجل"، جامعة

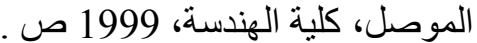

10. B.S. 1881: part 116 :1983, "Testing of Hardened Concrete", British Standard Institution, 1983.

11. ASTM C496-04, "Splitting Tensile Strength of Cylindrical Concrete Speciemens", American Society for Testing and Materials, 2004.

12. ASTM C143-04 "Slump of Hydraulic-Cement Concrete", American Society for Testing and Materials, 2004.

13. Benachour, Y., Davy, C.A., Skoczylas, F., and Houari, H., "Effect of a high calcite filler addition upon microstructural, mechanical, shrinkage and transport properties of a mortar", Cement and Concrete Research, Vol.38, 2008,PP. 727-736.

14. Celik,T., and Marar,K., "Effects of crushed stone dust on some properties of concrete", Cement and Concrete Research, Vol.26, 1996, PP.1121-1130.

15. الاوجار، إيمان قاسم يحبى "تحضير وتقييم ركام خفيف الوزن من مواد خام محلية ودراسة تاثير بعض المضافات"

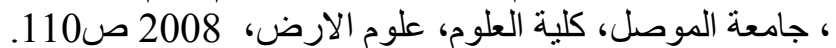

16. Rattachan, S., and Lorprayoon, C.," Korat clays as raw materials for light weight aggregates", Science Asia, Vol.31, 2005, PP. 277-281.

$$
\text { تم اجراء البحث في كلية ألهنسة = جامعة ألموصل }
$$

